\title{
The value of $\mathrm{X}$-ray digital tomosynthesis in the diagnosis of urinary calculi
}

\author{
SHIFENG LIU ${ }^{1}$, HONG WANG $^{2}$, WEIHUA FENG ${ }^{3}$, XIAOKUN HU $^{1}$, JIAN GUO $^{3}$, \\ QINGJUN SHANG ${ }^{4}$, ZIXIANG LI ${ }^{1}$ and HONGSHENG YU ${ }^{5}$ \\ ${ }^{1}$ Center for Interventional Medicine; Departments of ${ }^{2}$ Dermatology, ${ }^{3}$ Radiology, ${ }^{4}$ Tumor Radiotherapy, and \\ ${ }^{5}$ Tumor-Comprehensive Treatment, The Affiliated Hospital of Qingdao University, Qingdao, Shandong 266555, P.R. China
}

Received May 17, 2017; Accepted September 7, 2017

DOI: $10.3892 /$ etm.2017.5531

\begin{abstract}
Urinary calculus is a common and recurrent condition that affects kidney function. The present study evaluated the use of digital tomosynthesis (DTS) and Kidneys-Ureters-Bladder (KUB) radiography as methods of diagnosing urinary calculi. Unenhanced multidetector computed tomography (UMDCT) was used in the diagnosis of calculi. KUB radiography and DTS procedures were conducted on patients prior to and following bowel preparation to detect kidney, ureteral and bladder calculi. Differences in diagnostic performance of KUB radiography and DTS imaging on prepared and unprepared bowel were evaluated using the $\chi^{2}$ test. The consistency of diagnostic results between two examining physicians was analyzed using the $\kappa$ test. A total of 138 calculi from 80 patients were detected via UMDCT. The calculi detection rates of KUB prior to and following bowel preparation were 47.8 and $66.7 \%$ respectively, and the calculi detection rate of DTS prior to and following bowel preparation were 94.2 and $96.4 \%$, respectively. The detection rates of calculi $>5 \mathrm{~mm}$ via KUB prior to and following bowel preparation were 56.6 and $73.5 \%$ respectively, and in DTS they were $100 \%$ prior to and following bowel preparation. Economically, DTS performed on the unprepared bowel was the most cost effective, followed by DTS on the prepared bowel, KUB on the unprepared bowel and KUB on the prepared bowel. Therefore, the current study concluded that DTS may be an appropriate first-line imaging technique in patients with urinary calculi.
\end{abstract}

Correspondence to: Dr Hongsheng Yu, Department of Tumor-Comprehensive Treatment, The Affiliated Hospital of Qingdao University, 1677 Wutaishan Road, Huangdao, Qingdao, Shandong 266555, P.R. China

E-mail: hongshengyu012@163.com

Key words: urinary calculi, computer tomography, digital tomosynthesis, kidneys-ureters-bladder, unenhanced multidetector computed tomography

\section{Introduction}

Urinary calculus is a common disease that has a high recurrence rate and the age of patients at diagnosis is decreasing $(1,2)$. Thus, it is important to establish an effective and accurate diagnostic method to ensure that appropriate treatment is administered. The Kidneys-Ureters-Bladder (KUB) radiograph is able to effectively identify the location of calculi (3). However, due to the influence of intestinal gas and the overlapping area of the abdominal cavity, KUB radiography has certain disadvantages, including low-density resolution, low sensitivity and low accuracy (4). Unenhanced multidetector computed tomography (UMDCT) is the procedure of choice for the radiological evaluation of patients with renal colic (5). UMDCT is widely used in the differential diagnosis of urinary calculi due to its speed, convenience and high accuracy (6). However, the radiation dose and cost of UMDCT is high and UMDCT is unable to evaluate renal function. The development of digital tomosynthesis (DTS) has received a lot of attention as it administers a low dose of radiation and has a high resolution (7-10). DTS technology is widely used within dentistry and orthopedics as well as in the imaging of the breast, chest and blood vessels $(11,12)$. However, few studies have been performed to assess the application of DTS on the gastrointestinal and urinary tracts. The present study compared the rate of calculi detection via KUB radiography and DTS prior to and following bowel preparation in 80 patients with urinary calculi confirmed by UMDCT. The efficacy of DTS in the diagnosis of patients with urinary calculi was therefore assessed.

\section{Patients and methods}

Patients. A total of 80 patients were selected from The Affiliated Hospital of Qingdao University between June 2013 and June 2015. The sample consisted of 50 males and 30 females with an average age of 50 years (range, 14-80 years). All patients involved in the study had a history of lower back pain; 63 had experienced renal buckle pain, 43 had suffered from hematuria and 5 patients had a history of dysuria post-exercise. Additionally, 77 patients with hematuria were diagnosed. Out of these patients, 38 were diagnosed via microscopic examination, 18 through the naked eye and 21 via positive urine leucocyte samples. From the 80 included patients, 138 calculi were detected via UMDCT. 
Post-diagnosis, all patients received extracorporeal ultrasonic lithotripsy combined with medical expulsive therapy as treatment. The present study was approved by the hospital ethics committee of the Affiliated Hospital of Qingdao University and all patients provided their informed consent.

Inspection methods. The flat plate multi-function digital Perspective Photography System (Sonialvision Safire II, Shimadzu Co., Kyoto, Japan) with post-image processing and X-ray digital radiography (DR; Ysio, Siemens Healthineers, Erlangen, Germany) were used in this study for basic imaging.

Patients initially received a plain X-ray, a KUB radiograph and DTS for imaging. For bowel preparation, patients drank 11 warm water containing one packet of polyethylene glycol electrolyte powder $(68.56 \mathrm{~g} / \mathrm{l})$ in the evening, followed by the oral consumption of $40 \mathrm{ml}, 50 \%$ magnesium sulfate the next morning. Patients then underwent plain X-rays, KUB radiography and DTS.

UMDCT was used in the diagnosis of calculi. KUB radiography and DTS procedures were conducted on patients prior to and following bowel preparation to detect kidney, ureteral and bladder calculi. KUB radiograph inspection extended from the xiphoid process to the pubic symphysis, and examined the kidneys, ureters and bladder. At the time of DTS examination, the probe center was directed towards the upper ureteral horizontal station and scanned in wide scope whilst patients stood upright and held their breath. Images were processed using a layer thickness of 2-5 mm, a height of 130-140 mm (from the center level of the probe to the examining table) and a $150 \mathrm{~mm}$ scope of reconstruction. KUB radiography and DTS imaging were performed and examined by two physicians who were aware of patient medical history, but unaware of their identity and UMDCT results. MIP is a post processing technique that takes the highest-attenuation voxel in a predetermined slab of data and projects it from the user toward the viewing screen, resulting in a two-dimensional image.

Radiation dose. The entrance skin dose (ESD) for KUB radiographs and DTS imaging was estimated using RGD-3B thermoluminescent dosimeters (TLDs; The Chinese People's Liberation Army Chemical Defense Research Institute, Beijing, China). Briefly, the TLD detector was wrapped with black paper and double adhesive tape was attached. The TLDs detector was adhered to the skin surface in the center of the field of vision. At the end of examination, the dose area product (DAP), exposure parameters, including $\mathrm{kV}$ and $\mathrm{mAs}$, and TLDs numbers were recorded.

Dose-area product (DAP) was measured using a DAP meter (IBA USA, Reston, VA, USA). Effective doses (ED) were calculated from DAP values using RefDose software (VD0010135; IBA, San Francisco, CA, USA). The results from UMDCT were recorded, including the CTDlvol [CT dose index $(\mathrm{mGy})$ ] and DLP [dose length product $(\mathrm{mGy} \mathrm{cm})$ ]. EDs were calculated using the equation: $\mathrm{ED}=\mathrm{DLP} \times \mathrm{K}$, with $\mathrm{K}$ representing conversion factors. The $\mathrm{k}$ coefficient for the abdomen and pelvis was 0.015 (13).

Cost effectiveness. Following a study reported by Moores, the cost effectiveness was calculated as follows: Cost effectiveness $(\%)=($ inspection fee $\mathrm{x}$ case)/positive detection rate (14).
Statistical analysis. Based on the results of UMDCT, the sensitivity of DTS imaging and KUB radiography prior to and following bowel preparation for urinary calculi diagnosis was determined. The data were analyzed using SPSS 17 software (SPSS, Inc., Chicago, IL, USA). The $\chi^{2}$ test was used to calculate the difference in diagnostic capability of the four tested methods and $\mathrm{P}<0.05$ was considered to indicate a statistically significant difference. The consistency of diagnostic results was analyzed using Cohen's $\kappa$ test. $\kappa \geq 0.75$ was considered to be satisfactory.

\section{Results}

Diagnostic imaging methods. A total of 138 calculi were identified via UMDCT. These included 52 cases of renal calculi (20 patients with calculi $<5 \mathrm{~mm}, 32$ with calculi $>5 \mathrm{~mm}$ ), 83 cases of ureteral calculi ( 35 patients with calculi $<5 \mathrm{~mm}, 48$ with calculi $>5 \mathrm{~mm}$ ) and 3 cases of bladder calculi ( 3 patients with calculi $>5 \mathrm{~mm}$ ) (Table I). The detection rates of calculi $>5 \mathrm{~mm}$ via KUB radiography and DTS prior to bowel preparation were 56.6 and $73.5 \%$ respectively and were all $100 \%$ following bowel preparation, respectively. Among the four diagnostic methods, the $\kappa$ values that determined diagnostic consistency between the two examining physicians were 0.76 , $0.78,0.85$, and 0.85 respectively.

Effective radiation doses. The ED of DTS was $0.90 \mathrm{mSv}$. KUB radiography had an ED of $0.59 \mathrm{mSv}$ and UMDCT had an ED of $2.68 \mathrm{mSv}$. The differences in ED between each diagnostic method were all $\mathrm{P}<0.05$ (Table II).

Detection rates. The detection rates of all calculi via KUB radiography prior to and following bowel preparation were 47.8 and $66.7 \%$, respectively. The detection rates of calculi via DTS prior to and following bowel preparation were 94.2 and 96.4\%, respectively (Table III).

Cost effectiveness. The total cost of KUB prior to bowel preparation or following bowel preparation were cheaper than DTS prior to bowel preparation or following bowel preparation. From high to low, the cost effectiveness for each method was as follows: DTS prior to bowel preparation, DTS following bowel preparation, KUB prior to bowel preparation and KUB following bowel preparation (Table III).

Diagnostic sensitivities. The diagnostic sensitivities of KUB radiography and DTS prior to and following bowel preparation of calculi $<5 \mathrm{~mm}$ and $>5 \mathrm{~mm}$ were compared. There was no significant difference in the sensitivity of DTS diagnosis when conducted on the prepared bowel compared with the unprepared bowel. All other differences were considered statistically significant (all $\mathrm{P}<0.05$, Table IV). Additionally, Figs. 1 and 2 detail the difference in calculi detection between each imaging method. UMDCT, CT and DTS images coupled with KUB radiographs from a 31-year-old male patient diagnosed with urinary tract calculi are presented in Fig. 1. UMDCT, CT and DTS images coupled with KUB radiographs of a 55-year-old male patient diagnosed with urinary tract calculi are presented in Fig. 2. DTS and UMDCT clearly show urinary calculi. However, some of the stones are not clear with KUB. 
Table I. Number of calculi $>5 \mathrm{~mm}$ and $<5 \mathrm{~mm}$ detected in the kidney, ureters and bladder via KUB radiography and DTS used with and without BP.

\begin{tabular}{|c|c|c|c|c|c|c|c|c|c|}
\hline \multirow[b]{2}{*}{$\begin{array}{l}\text { Examination } \\
\text { method }\end{array}$} & \multicolumn{4}{|c|}{ Calculi $<5 \mathrm{~mm}(\mathrm{n}=55)$} & \multicolumn{4}{|c|}{ Calculi $>5 \mathrm{~mm}(\mathrm{n}=83)$} & \multirow[b]{2}{*}{ Total } \\
\hline & $\begin{array}{l}\text { Kidney } \\
(n=40)\end{array}$ & $\begin{array}{l}\text { Ureteral } \\
(n=15)\end{array}$ & $\begin{array}{l}\text { Bladder } \\
\quad(n=0)\end{array}$ & Sum & $\begin{array}{l}\text { Kidney } \\
(n=12)\end{array}$ & $\begin{array}{l}\text { Ureteral } \\
(n=68)\end{array}$ & $\begin{array}{c}\text { Bladder } \\
(n=3)\end{array}$ & Sum & \\
\hline KUB without BP & 13 & 5 & 0 & 18 & 8 & 38 & 2 & 48 & 66 \\
\hline KUB with BP & 23 & 8 & 0 & 31 & 11 & 47 & 3 & 61 & 92 \\
\hline DTS without BP & 32 & 15 & 0 & 47 & 12 & 68 & 3 & 83 & 130 \\
\hline DTS with BP & 35 & 15 & 0 & 50 & 12 & 68 & 3 & 83 & 133 \\
\hline
\end{tabular}

KUB, kidneys-ureters-bladder; DTS, digital tomosynthesis; BP, bowel preparation.

Table II. Analysis of KUB radiography, DTS and UMDCT effective doses.

\begin{tabular}{lcrr}
\hline Comparison of diagnostic methods & Effective dose comparison & t value & P-value \\
\hline KUB vs. UMDCT & 0.59 vs. 2.68 & 306.78 & P $<0.05$ \\
DTS vs. UMDCT & 0.90 vs. 2.68 & 242.69 & P $<0.05$ \\
KUB vs. DTS & 0.59 vs. 0.90 & 34.51 & $P<0.05$ \\
\hline
\end{tabular}

KUB, kidneys-ureters-bladder; DTS, digital tomosynthesis; UMDCT, unenhanced multidetector computed tomography.

Table III. Cost effectiveness of diagnostic methods.

\begin{tabular}{lccc}
\hline Examination method & Total cost (Renminbi) & Detection rate (\%) & Cost effectiveness $(\%)$ \\
\hline KUB prior to bowel preparation & 7,200 & 47.8 & 150.6 \\
KUB following bowel preparation & 11,200 & 66.7 & 167.9 \\
DTS prior to bowel preparation & 9,600 & 94.2 & 101.9 \\
DTS with bowel preparation & 13,600 & 96.4 & 141.1 \\
UMDCT & 32,000 & 100 & 320 \\
\hline
\end{tabular}

KUB, kidneys-ureters-bladder; DTS, digital tomosynthesis; UMDCT, unenhanced multidetector computed tomography.

Table IV. Differences in diagnostic sensitivity of KUB radiography and DTS with and without bowel preparation on calculi $>5 \mathrm{~mm}$ and $<5 \mathrm{~mm}$.

\begin{tabular}{|c|c|c|c|c|c|c|}
\hline \multirow[b]{2}{*}{ Method comparison } & \multicolumn{2}{|c|}{ All calculi } & \multicolumn{2}{|c|}{ Calculi $<5 \mathrm{~mm}$} & \multicolumn{2}{|c|}{ Calculi $>5 \mathrm{~mm}$} \\
\hline & $\chi^{2}$ value & P-value & $\chi^{2}$ value & P-value & $\chi^{2}$ value & P-value \\
\hline 1 vs. 2 & 10.007 & 0.002 & 6.219 & 0.013 & 4.515 & 0.034 \\
\hline 1 vs. 3 & 72.098 & 0.000 & 31.627 & 0.000 & 44.351 & $<0.000$ \\
\hline 1 vs. 4 & 80.856 & 0.000 & 39.440 & 0.000 & 44.351 & $<0.000$ \\
\hline 2 vs. 3 & 33.245 & 0.000 & 11.282 & 0.001 & 25.361 & $<0.000$ \\
\hline 2 vs. 4 & 40.432 & 0.000 & 16.905 & 0.000 & 25.361 & $<0.000$ \\
\hline 3 vs. 4 & 0.727 & 0.394 & 0.785 & 0.376 & - & - \\
\hline
\end{tabular}

Method 1, KUB prior to bowel preparation; Method 2, KUB following bowel preparation; Method 3, DTS prior to bowel preparation; Method 4, DTS following bowel preparation. DTS detected all calculi $(n=83)$, such that the data cannot be calculated statistically. KUB, kidneys-ureters-bladder; DTS, digital tomosynthesis . 

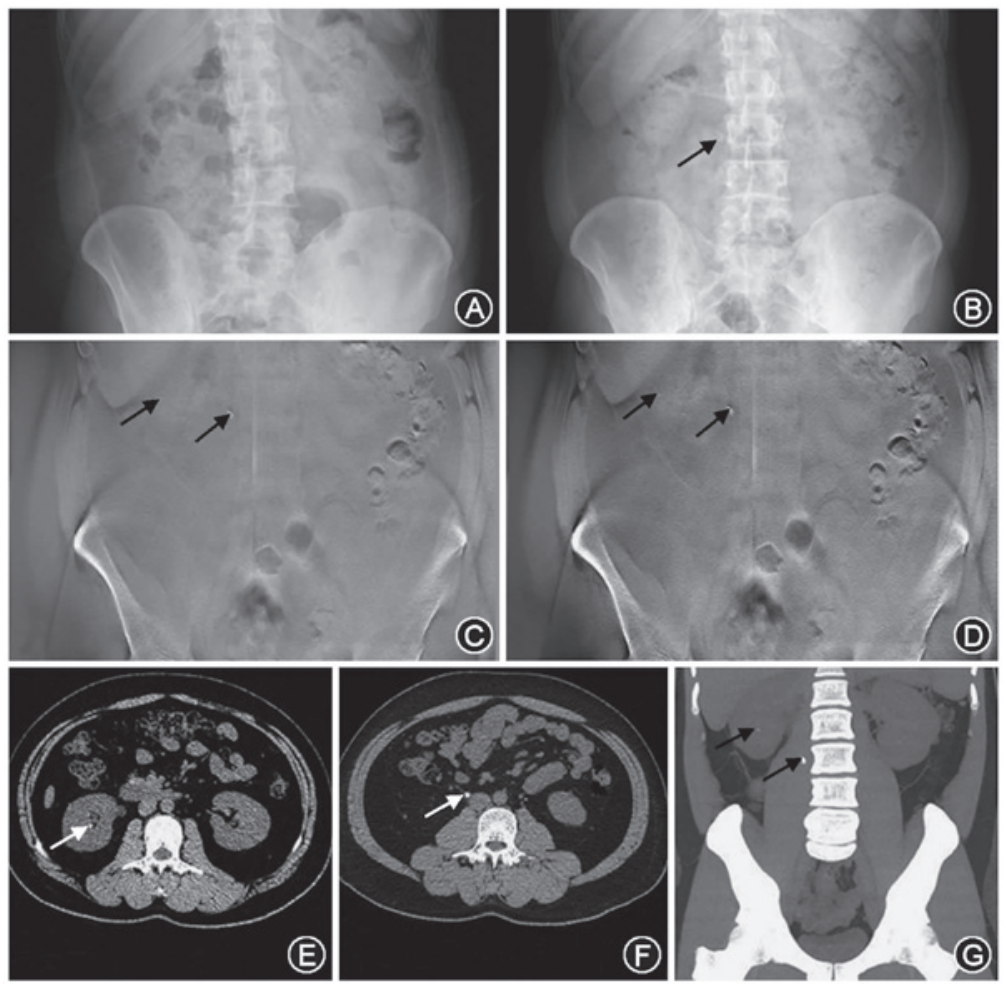

Figure 1. UMDCT, CT and DTS images coupled with KUB radiographs from a 31-year-old male patient diagnosed with urinary tract calculi. (A) No calculi were detected from the KUB radiograph on the unprepared bowel. (B) KUB radiography on prepared bowel detected a calculus on the right ureter (black arrow). DTS detected a right ureter and a right kidney calculus (C) prior to and (D) following bowel preparation (black arrows). (E) CT images detected a right kidney calculus (white arrow). (F) CT images detected a right ureter calculus (white arrow). (G) An MRP MIP reconstruction image of UMDCT detected a right kidney and right ureter calculus (white arrow). UMDCT, unenhanced multidetector computed tomography; CT, computed tomography; DTS, digital tomosynthesis; KUB, kidneys-ureters-bladder.
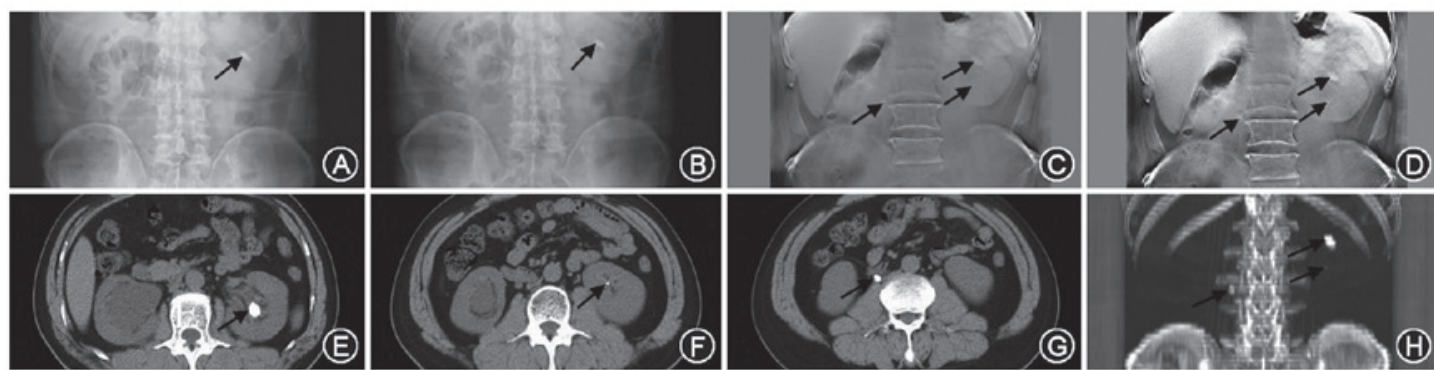

Figure 2. UMDCT, CT and DTS images coupled with KUB radiographs of a 55 year old male patient, diagnosed with urinary tract calculi. KUB detected a left kidney calculus (A) prior to and (B) following bowel preparation (black arrows). DTS detected a right ureter and left kidney calculus (C) prior to and (D) following bowel preparation (black arrow). (E) CT images detected a calculus in the upper portion of the left kidney (black arrow). (F) CT images detected a calculus in the lower portion of the left kidney (black arrow). (G) CT images detected a right ureteral calculus (black arrow). (H) MIP reconstruction image of UMDCT identified a left kidney and a left ureteral calculus (black arrows). UMDCT, unenhanced multidetector computed tomography; CT, computed tomography; DTS, digital tomosynthesis; KUB, kidneys-ureters-bladder.

\section{Discussion}

DTS imaging differs from traditional X digital photography; it is possible to obtain 74 high-definition images and a layer of original image from only one scanning (15). The displacement and superposition methods are used for image reconstruction, so that the image at the coronary level may obtained for any region of interest (16). DTS can also accurately and clearly detect the shape and contour of the kidney and image fine structures, including the renal pelvis, to more clearly show the morphology of lesions at the edge of the longitudinal and changes in adjacent structures.
KUB radiography is currently the examination method of choice for patients with suspected urinary calculi, as it is simple to perform. However, its value as a diagnostic tool is limited as its detection sensitivity is relatively low, ranging between 58 and $62 \%(7,17)$. The low detection rates and low-density resolution associated with KUB radiography are primarily due to intestinal overlap. In the present study, the use of bowel preparation increased the DTS detection rate from 47.8 to $66.7 \%$. No significant differences were identified in the detection rate of DTS prior to and following bowel preparation for $>5 \mathrm{~mm}$ urinary calculi. There were few observable differences between these images, indicating that 
DTS was not affected by intestinal gas or observer experience, whereas the cost of DTS following bowel preparation was greater than that of DTS prior to bowel preparation. The total cost of KUB was cheaper than DTS; however, the detection rate and cost-effectiveness of KUB was no better compared with DTS. In addition, the detection rate of DTS was greater for urinary calculi $>5 \mathrm{~mm}$.

UMDCT has a reported sensitivity of $\sim 97 \%$ and a CT image reconstruction sensitivity of $99-100 \%$, making it the most sensitive diagnostic technique in patients with acute urinary calculi $(18,19)$. However, it administers a high dose of radiation to patients and follow-up is expensive, thus limiting its application. Previous studies determined that the conventional and low effective doses of UMDCT are higher than those of DTS (4,8-10). In the present study it was determined that the detection rate of DTS prior to bowel preparation was $94.2 \%$. Compared with UMDCT, the difference in the detection rate was insignificant, demonstrating that the diagnostic capabilities of the two techniques were similar. The effective dose of DTS is lower than that of UMDCT and it was determined to be more cost effective. Thus, it was determined that DTS is a more economical and effective method of detecting urinary calculi than UMDCT.

The application of DTS in the diagnosis of urinary calculi has been assessed. Mermuys et al (7) determined that the diagnostic performance of abdominal DTS was greater than that of planar digital radiography; DTS improved inter-reader reproducibility following a small increase in the radiation dose. However, little improvement was demonstrated in the diagnosis of ureteral calculi (7). In the current study, the radiologists knew the history of the patients, which may have been conducive to a high detection rate of calculi by DTS. Furthermore, differences in the diagnosis rates between two observers and the EDs of the different diagnostic methods were consistent with those from previous studies. In addition, the present study assessed the effect of intestinal preparation on the detection rate and the cost effectiveness of different diagnostic methods. DTS performed on the unprepared bowel was the most cost effective, which may be an appropriate first-line imaging technique in patients with urinary calculi.

There were a few limitations of the current study. Only a short-term follow-up was provided for included patients involved and did not include the use of DTS. Furthermore, the diagnosis of calculi was also not confirmed by surgery. Finally, the sample size of the current study was small.

In conclusion, the results of the current study demonstrated that urinary calculi were more efficiently diagnosed using abdominal DTS than conventional KUB. The radiation dose of DTS was lower and the detection rate was similar to that of UMDCT. Bowel preparation did not significantly affect rates of diagnosis. Thus, the results of the present study indicated that DTS may replace KUB as the first-line imaging technique in patients with suspected urinary calculi. It may also replace KUB radiography as the routine technique for calculi and extracorporeal gravel positioning as well as the method for monitoring the course of lithotripsy. However, further research using a larger sample size is required to determine this.

\section{References}

1. Sujatha D, Singh K, Vohra M, Kumar KV and Sunitha S: Antilithiatic activity of phlorotannin rich extract of sarghassum wightii on calcium oxalate urolithiais-in vitro and in vivo evaluation. Int Braz J Urol 41: 511-520, 2015.

2. Dursun M, Ozbek E, Otunctemur A, Sahin S and Cakir SS: Clinical presentation of urolithiasis in older and younger population. Arch Ital Urol Androl 86: 249-252, 2014.

3. Gürel K, Gürel S, Kalfaoğlu M, Yilmaz O and Metin A: Does an extra kidney-ureter-bladder radiograph taken in the upright position during routine intravenous urography provide diagnostic benefit? Diagn Interv Radiol 14: 205-211, 2008.

4. Wells IT, Raju VM, Rowberry BK, Johns S, Freeman SJ and Wells IP: Digital tomosynthesis-a new lease of life for the intravenous urogram? Br J Radiol 84: 464-468, 2011.

5. Lauritsen J, Andersen JR, Nordling J and Thomsen HS: Unenhanced computed tomography in acute renal colic reduces cost outside radiology department. Acta Radiol 49: 1182-1186, 2008.

6. Demirel A and Suma S: The efficacy of non-contrast helical computed tomography in the prediction of urinary stone composition in vivo. J Int Med Res 31: 1-5, 2003

7. Mermuys K, De Geeter F, Bacher K, Van De Moortele K, Coenegrachts K, Steyaert L and Casselman JW: Digital tomosynthesis in the detection of urolithiasis: Diagnostic performance and dosimetry compared with digital radiography with MDCT as the reference standard. AJR Am J Roentgenol 195: 161-167, 2010.

8. Neisius A, Astroza GM, Wang C, Nguyen G, Kuntz NJ, Januzis N, Ferrandino MN, Yoshizumi TT, Preminger GM and Lipkin ME: Digital tomosynthesis: A new technique for imaging nephrolithiasis. Specific organ doses and effective doses compared with renal stone protocol noncontrast computed tomography. Urology 83: 282-287, 2014.

9. Yan H, Godfrey DJ and Yin FF: Fast reconstruction of digital tomosynthesis using on-board images. Med Phys 35: 2162-2169, 2008.

10. Astroza GM, Neisius A, Wang AJ, Nguyen G, Toncheva G, Wang C, Januzis N, Lowry C, Ferrandino MN, Neville AN, et al: Radiation exposure in the follow-up of patients with urolithiasis comparing digital tomosynthesis, non-contrast $\mathrm{CT}$, standard KUB, and IVU. J Endourol 27: 1187-1191, 2013.

11. Galea A, Dubbins P, Riordan R, Adlan T, Roobottom C and Gay D: The value of digital tomosynthesis of the chest as a problem-solving tool for suspected pulmonary nodules and hilar lesions detected on chest radiography. Eur J Radiol 84: 1012-1018, 2015.

12. Aoki T, Fujii M, Yamashita Y, Takahashi H, Oki H, Hayashida Y, Saito K, Tanaka Y and Korogi Y: Tomosynthesis of the wrist and hand in patients with rheumatoid arthritis: Comparison with radiography and MRI. AJR Am J Roentgenol 202: 386-390, 2014.

13. Moores BM: Cost-risk-benefit analysis in diagnostic radiology: A theoretical and economic basis for radiation protection of the patient. Radiat Prot Dosimetry 169: 2-10, 2016.

14. Landis JR and Koch GG: The measurement of observer agreement for categorical data. Biometrics 33: 159-174, 1977.

15. Liu S, Guo J, Hu X, Zhang H, Shang Q, Xu W and Feng W: Comparative study of X-ray digital DTS imaging and kidney ureter bladder radiography in urinary calculi. Zhonghua Yi Xue Za Zhi 95: 2010-2013, 2015 (In Chinese).

16. Dobbins JT III and Godfrey DJ: Digital x-ray tomosynthesis: Current state of the art and clinical potential. Phys Med Biol 48: R65-R106, 2003.

17. Yap WW, Belfield JC, Bhatnagar P, Kennish S and Wah TM: Evaluation of the sensitivity of scout radiographs on unenhanced helical CT in identifying ureteric calculi: A large UK tertiary referral centre experience. Br J Radiol 85: 800-806, 2012.

18. Park SJ, Yi BH, Lee HK, Kim YH, Kim GJ and Kim HC: Evaluation of patients with suspected ureteral calculi using sonography as an initial diagnostic tool: How can we improve diagnostic accuracy? J Ultrasound Med 27: 1441-1450, 2008.

19. Kwon JK, Chang IH, Moon YT, Lee JB, Park HJ and Park SB: Usefulness of low-dose nonenhanced computed tomography with iterative reconstruction for evaluation of urolithiasis: Diagnostic performance and agreement between the urologist and the radiologist. Urology 85: 531-538, 2015. 